\title{
Colangiopancreatografia retrógrada endoscópica por coledocolitíase no Hospital Universitário Walter Cantídio - UFC - avaliação de 56 casos
}

\section{Endoscopic retrograde cholangiopancreatography by coledocolitiase in the University Hospital Walter Cantidio - UFC - evaluation of 56}

\section{cases}

Diego da Costa Matos ${ }^{1}$. Paulo Roberto Veras Tavares ${ }^{1}$. Gustavo Melo Benevides ${ }^{2}$. Leonardo José Sales da Costa $^{2}$. Fred Olavo Andrade Aragão Carneiro ${ }^{2}$. Miguel Ângelo Nobre e Souza ${ }^{1,2}$. Marcellus Henrique Loiola Ponte de Souza ${ }^{1,2}$.

1 Universidade Federal do Ceará (UFC), Fortaleza, Ceará, Brasil. 2 Hospital Universitário Walter Cantídio (HUWC), Fortaleza, Ceará, Brasil.

\section{RESUMO}

A coledocolitíase é a causa mais frequente de obstrução biliar comumente vista na prática gastroenterológica. Objetivo: avaliar taxa e fatores relacionados ao sucesso assim como complicações nos pacientes com coledocolitíase, encaminhados para CPRE no HUWC-UFC. Metodologia: Estudo retrospectivo de 56 pacientes submetidos a 67 CPREs por coledocolitíase de 01/2017 a 04/2018. As informações foram retiradas do banco de dados da unidade de Endoscopia e prontuários. Analisadas as taxas de sucesso (retirada completa dos cálculos), complicações (pancreatite, hemorragia e perfuração) e fatores relacionados a estes desfechos. CEP: 43321014.6.0000.5045. Resultados: Houve predominância de mulheres (80\%), com idade média de 51 anos. A taxa sucesso foi $89 \%$. Com complementação terapêutica cirúrgica em $9 \%$. 2\% dos casos, estão ainda em seguimento ambulatorial. A complicação mais frequente foi pancreatite (7\%), sendo (1) paciente com evolução ao óbito (1,8\%). Sem a existência de perfurações ou hemorragias. Houve associação entre a presença de cálculos grandes $(\mathrm{p}=0.05)$ e desproporção da via biliar $(\mathrm{p}=0.01)$ com insucesso da CPRE. Não houve nenhuma associação dos fatores avaliados com a presença de complicações. Conclusão: $\mathrm{O}$ estudo demonstra que houve alta taxa de sucesso, como descrito na literatura, sendo a presença de cálculos grandes e desproporção fatores relacionados ao insucesso da CPRE.

Palavras-chave: Coledocolitíase. Colangiopancreatografia retrógrada endoscópica. Pancreatite.

\section{ABSTRACT}

Choledocholithiasis is the most frequent cause of biliary obstruction commonly seen in gastroenterological practice. Objective: evaluate the rate and factors related to success and complications in patients with choledocholithiasis, referred or ERCP in HUWCUFC. Methodology: Retrospective study of 56 patients submitted to 67 examinations for choledocholithiasis from 01/2017 to 04/2018. The information was taken from the Endoscopy unit medical records database. Success rates (complete withdrawal of calculus), complications (pancreatitis, hemorrhage and perforation), and factors related to these outcomes were analyzed. CEP: 43321014.6.0000.5045. Results: There was a predominance of women (80\%), in their early 50 years old. The success rate was $89 \%$. With surgical therapeutic complementation in $9 \% .2 \%$ of the cases are still under follow-up. The most frequent complication was pancreatitis (7\%), being (1) patient with evolution to death $(1.8 \%)$ without perforations or bleeding. There was an association between the presence of large stones $(p=0.05)$ and bile duct disproportion $(p=0.01)$ with ERCP failure. There was no association of the factors evaluated, with the presence of complications. Conclusion: The study shows that there was a high success rate, as described in the literature. The presence of large stones and disproportion, factors related to ERCP failure.

Keywords: Choledocholithiasis. Cholangiopancreatography, endoscopic retrograde. Pancreatitis.

Autor correspondente: Diego da Costa Matos, Rua Capitão Francisco Pedro, 1016, Rodolfo Teófilo, Fortaleza, Ceará, Brasil. CEP: 60430-372. Telefone: +55 85 99636-6634. E-mail: diegodacostamatos@gmail.com

Conflito de interesses: Não há qualquer conflito de interesses por parte de qualquer um dos autores.

Recebido em: 20 Fev 2019; Revisado em: 12 Abr 2019; Aceito em: 04 Mai 2019. 


\section{INTRODUÇÃO}

A coledocolitíase ou litíase no ducto biliar comum é a causa mais frequente de obstrução biliar e muito comumente vista na prática gastroenterológica. Aproximadamente 5 a $22 \%$ da população ocidental possui litíase vesicular, ${ }^{1}$ apresentando um marcado acréscimo na incidência entre os 35 e 55 anos e aumentando gradualmente com o aumento da idade, é mais frequente nas mulheres. ${ }^{2}$ A coledocolitíase incide em 5 a $10 \%$ dos casos de litíase vesicular. ${ }^{1}$

No Brasil, a incidência desse tipo de obstrução das vias biliares está entre 8 a 10\%. Nos dias atuais, após o advento da cirurgia laparoscópica com a indicação mais precoce, há relatos de que a incidência de coledocolitíase tenha diminuído para níveis de $3 \%$ a $6 \%{ }^{1,3,4}$ A grande maioria desses cálculos forma-se na vesícula biliar e migra para o colédoco através do ducto cístico. ${ }^{5}$ Estes cálculos são chamados de cálculos secundários, já os que se formam no próprio colédoco sendo chamados de cálculos primários. A grande maioria dos cálculos presentes no hepatocolédoco são pequenos e provenientes da vesícula biliar. ${ }^{2}$

Estes cálculos podem passar para o duodeno espontaneamente, ou impactar-se na papila, provocando obstrução biliar, colangite e pancreatite biliar. ${ }^{5}$ Em geral esses eventos adversos são sérios e podem ser uma ameaça a vida dos pacientes, justificando que na presença de coledocolitíase, seja necessária alguma intervenção terapêutica para remoção dos cálculos. ${ }^{3}$

A CPRE foi primeiramente relatada em 1968 e rapidamente aceita como uma técnica segura e de avaliação direta das doenças pancreatobiliares. Com a introdução da papilotomia (esfincterotomia) endoscópica em 1974, se desenvolveu a endoscopia terapêutica pancreatobiliar e tornou-se o método mais comum para extração de cálculos no ducto biliar comum..$^{6,7,8}$ A colangiopancreatografia endoscópica retrógrada (CPRE) com esfincterotomia é reconhecida como método endoscópico primário para extração dos cálculos e com alta taxa de sucesso na sua remoção, sendo superior a $90 \%, 3,4,6,7,9,10$ além ser responsável por baixos índice de complicações. ${ }^{4}$

Em aproximadamente 10 a $15 \%$ destes pacientes, os cálculos do ducto biliar serão difíceis de ser removidos por serem cálculos gigantes $(>15 \mathrm{~mm})$, múltiplos cálculos, acesso desafiador ao ducto biliar (divertículo periampular, billroth II, gastrojejunostomia em y-Roux), cálculos em forma de barril e tortuosidade e afilamento do ducto biliar comum distal, necessitando de procedimentos adicionais, ${ }^{1,4,6,7,10}$ que na maioria dos hospitais brasileiros vinculados ao SUS, não estão disponíveis em razão dos custos e da falta de financiamento para aquisição de novas tecnologias.

Neste estudo, pretendeu-se avaliar a taxa e os fatores relacionados ao sucesso, as complicações e a necessidade de procedimento cirúrgico adicional, nos pacientes com coledocolitíase, encaminhados para CPRE no HUWC- UFC.

\section{MATERIAIS E MÉTODOS}

Estudo retrospectivo de 56 pacientes submetidos a 67 exames no HUWC-UFC, no período de janeiro de 2017 a abril de 2018. Foram selecionados os pacientes submetidos a CPRE com diagnóstico de coledocolitíase como principal indicação. As informações foram colhidas através de bancos de dados da unidade de Endoscopia e prontuários. Foram analisadas as taxas de sucesso (retirada completa dos cálculos) e de complicações (pancreatite, hemorragia e perfuração), bem como os eventuais fatores relacionados a estes desfechos, como idade, gênero, tamanho do cálculo, número de cálculos, necessidade de dilatação da papila, presença de desproporção da via biliar distal (tamanho da via biliar menor que o tamanho do cálculo) e presença de divertículos. Para análise estatística foram usados o Graph Pad Prisma e o teste de Fisher. Aprovado pelo comitê de ética em pesquisa em seres humanos do HUWC (Protocolo: 43321014.6.0000.5045).

\section{RESULTADOS}

\section{Pacientes}

No período de janeiro de 2017 a abril de 2018, foram realizadas um total de 139 CPREs no Setor de Endoscopia do HUWC. Desses, 67 CPREs tiveram como principal indicação a coledocolitíase, totalizando 56 pacientes com o mínimo de idade de 4 anos e o paciente mais longevo com 92 anos. Em todos os pacientes houve sucesso no acesso as vias biliares. Do total de 56 pacientes, $44(80 \%)$ eram do sexo feminino. A média de idade foi de 51 anos. Foi observado o sucesso da retirada completa dos cálculos em 50 pacientes (89\%). Nos 6 pacientes (11\%) em que não houve o sucesso da retirada, $5(9 \%)$ foram submetidos a complementação terapêutica cirúrgica. Um paciente ainda está em seguimento $(2 \%)$.

\section{Fatores relacionado a taxa de insucesso}

A Tabela 1 mostra que a presença de cálculos maiores que $12 \mathrm{~mm}$ foi significativamente $(\mathrm{P}<0,05)$ mais presente no grupo de insucesso (50\%), quando comparado ou grupo de sucesso $(12 \%)$. Houve também diferença significativa $(\mathrm{P}<0,01)$ em relação a desproporção da via biliar distal no grupo insucesso (50\%), quando comparado com o grupo que teve sucesso no procedimento $(4 \%)$. Por outro lado, presença de múltiplos $(>1)$ cálculos, a idade, gênero, número de cálculos, necessidade de dilatação de papila, presença de divertículos não apresentaram diferenças entre o grupo com sucesso do procedimento em relação com o grupo insucesso do procedimento conforme demonstrado na Figura 1.

\section{Complicações}

A Tabela 2 e 3 apresenta a complicação mais frequente que foi a pancreatite pós CPRE em 4 pacientes (7\%), sendo 1 paciente com evolução para óbito $(1,8 \%)$. Não existiram perfurações ou hemorragias. Não houve nenhuma associação dos fatores avaliados com a presença ou ausência de complicações relacionadas ao procedimento. 
Tabela 1. Fatores relacionado a taxa de insucesso da CPRE para 56 pacientes com colecolitíase no HUWC-UFC.

\begin{tabular}{llll}
\hline Perfil pesquisado & $\begin{array}{l}\text { Sucesso } \\
\mathbf{N}=\mathbf{5 0}\end{array}$ & $\begin{array}{l}\text { Insucesso } \\
\mathbf{N = 6}\end{array}$ & P \\
\hline Idade $>\mathbf{5 0}$ & $54 \%(27)$ & $33,3 \%(2)$ & 0.3007 \\
Mulher & $78 \%(39)$ & $100 \%(6)$ & 0.1321 \\
Calculo $>\mathbf{1 2} \mathbf{~ m m}$ & $12 \%(6)$ & $50 \%(3)$ & $\mathbf{0 . 0 4 6 3}$ \\
Número $>\mathbf{1}$ & $52 \%(26)$ & $50 \%(3)$ & 0.6299 \\
Dilatação de papila & $30 \%(15)$ & $66,6 \%(4)$ & 0.0936 \\
Desproporção & $4 \%(2)$ & $50 \%(3)$ & $\mathbf{0 . 0 0 6 6}$ \\
Divertículo & $18 \%(9)$ & $16,6 \%(1)$ & 0.7107 \\
\hline
\end{tabular}

*Teste de Fisher.

Figura 1. Demonstrativo da taxa de sucesso e insucesso dos 56 pacientes com coledocolitíase em acompanhamento no HUWC-UFC.

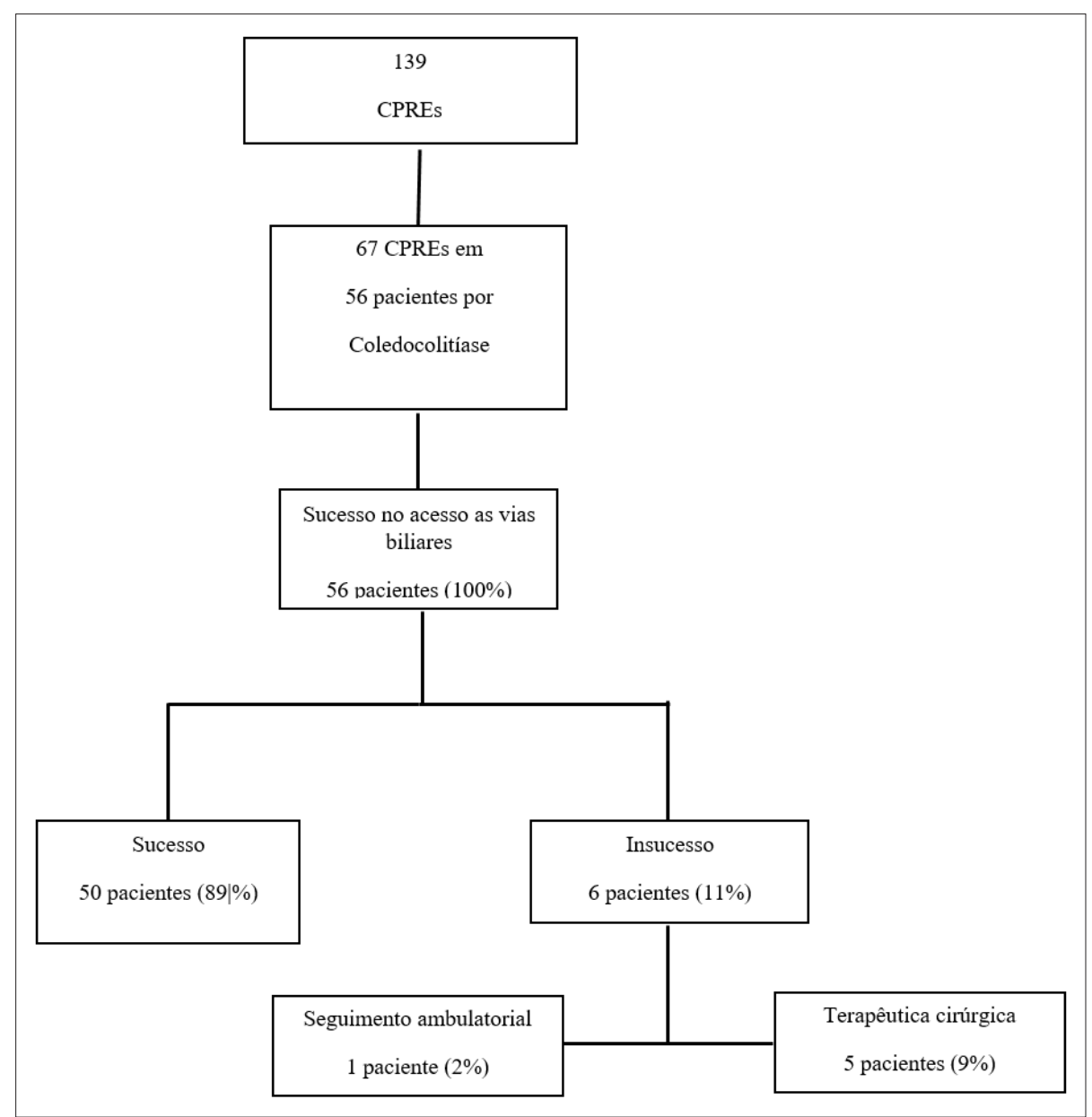

Tabela 2. Taxa de complicação das CPREs em 56 casos do HUWC-UFC.

\begin{tabular}{ll}
\hline Complicações & $\mathbf{N}=\mathbf{5 6}$ \\
\hline Pancreatite pós CPRE & $4(7 \%)$ \\
Hemorragia & 0 \\
Perfuração & 0 \\
Óbito & $1(1,8 \%)$ \\
\hline
\end{tabular}


Tabela 3. Fatores relacionados com a taxa de complicação da CPREs em 56 caso do HUWC-UFC.

\begin{tabular}{llll}
\hline & $\begin{array}{l}\text { Sem complicação } \\
\mathbf{N}=\mathbf{5 2}\end{array}$ & $\begin{array}{l}\text { Com complicação } \\
\mathbf{N = 4}\end{array}$ & $\mathbf{P}$ \\
\hline Idade $>\mathbf{5 0}$ & $50 \%(26)$ & $75 \%(3)$ & 0.3333 \\
Mulher & $83 \%(3)$ & $50 \%(2)$ & 0.1694 \\
Calculo $>\mathbf{1 2} \mathbf{~ m m}$ & $17 \%(9)$ & 0 & 0.4856 \\
Número $>\mathbf{1}$ & $56 \%(9)$ & $50 \%(2)$ & 0.6084 \\
Dilatação de papila & $35 \%(18)$ & $25 \%(1)$ & 0.5818 \\
Desproporção & $6 \%(3)$ & $75 \%(1)$ & 0.2673 \\
Divertículo & $19 \%(10)$ & 0 & 0.4443 \\
\hline
\end{tabular}

*Teste de Fisher.

\section{DISCUSSÃO}

A CPRE é um procedimento tecnicamente desafiador, com taxas relatadas de canulação malsucedida variando entre $3 \%$ e $10 \% .^{9}$ Em nosso estudo todos os pacientes obtiveram a canulação da via biliar principal. A pesquisa avaliou a taxa e os fatores relacionados ao sucesso e as complicações nos pacientes com coledocolitíase encaminhados para CPRE no HUWC, um hospital universitário do SUS. Através dele podemos constatar que houve uma alta taxa de sucesso e de complicação próximo ao descrito na literatura, sendo a presença de cálculos grandes e a desproporção da via biliar distal, fatores relacionados ao insucesso do procedimento. Não conseguimos definir fatores relacionados a complicações.

As amostras estudadas em sua maioria dos pacientes eram do sexo feminino (80\%) com idade superior a 50 anos (52\%). Corroborando com a literatura, estudos mostram que a incidência de colelitíase (destes 5 a $10 \%$ com coledocolitíase associada) ocorre entre os 35 e 55 anos e aumenta com a idade. $^{2}$

Dos 56 pacientes, a grande maioria obteve sucesso na extração completa do(s) cálculo(s) da via biliar, uma taxa de sucesso $89 \%$, conforme já descrito na literatura. A remoção dos cálculos de forma bem-sucedida é relatada na literatura em taxas que variam de $86-98 \%$ das tentativas, ${ }^{9}$ sendo a papilotomia endoscópica a técnica standard de acesso à via biliar principal para remoção de cálculos da mesma com uso de balão de extração ou cesto de Dormia. ${ }^{6,8}$

Em 19 pacientes deste estudo, realizaram a papilotomia endoscópica associada a dilatação papilar com balão de grande diâmetro. Comparando os grupos de sucesso com dilatação de papila (30\%) com o grupo de não dilatação da papila $(66,6 \%)$, não houveram diferença estatística para concluir que a associação do método melhorou a taxa de sucesso, discordando com o descrito na literatura. Ersoz et al (2003), descreveram pela primeira vez a dilatação papilar com balões de grande diâmetro $(>12 \mathrm{~mm})$ associada a papilotomia para remoção de cálculos difíceis da via biliar principal (cálculos grandes ou múltiplos cálculos), ${ }^{11}$ atualmente já estabelecida como uma técnica adjuvante a papilotomia que aumenta as taxas de sucesso na completa remoção de cálculos grandes e difíceis do ducto biliar comum com baixos índices de pancreatite pós CPRE (2,3\%). ${ }^{6,8,10,12}$ Acreditamos que a divergência entre os nossos resultados e os apresentados na literatura atual se deve ao número reduzido de pacientes na amostra.

Mais da metade dos pacientes encontraram-se mais de um cálculo. Nove (9) dos cinquenta e seis (56) pacientes, os cálculos eram grandes $(>12 \mathrm{~mm})$, comparando o grupo de insucesso ( 3 casos ou 50\%) em relação ao grupo de sucesso (6 casos ou 12\%), demonstrou essa variável ser um fator influenciador na taxa de insucesso $(\mathrm{p}<0.05)$. A desproporção entre o diâmetro do cálculo e o colédoco se apresentou em 5 casos, com 3 casos no grupo de insucesso, sendo mais um fator influenciador na taxa de insucesso $(p<0.01)$. O resultado corrobora com os achados na literatura, onde descreve que o manejo dos cálculos biliares pode ser difícil quando confrontados com grandes e/ou múltiplos cálculos, desproporção entre o tamanho do cálculo e o tamanho do ducto biliar comum distal ou da papilotomia e do ducto biliar comum distal. ${ }^{6,7,9} \mathrm{Em} \mathrm{dez}$ (10) pacientes a papila se encontrava em posição peri ou intradiverticular onde somente um (1) dos casos no grupo de insucesso, não havendo diferença estatística em relação ao grupo de sucesso, discordando com a literatura.

O sucesso da CPRE também pode variar dependendo de fatores anatômicos, como a localização da papila adjacente ao divertículo e a anatomia modificada cirurgicamente. ${ }^{6,7,9} \mathrm{~A}$ minoria dos pacientes deste estudo não obtiveram sucesso na extração do (s) cálculo (s) em um ou mais procedimentos, encaminhando 5 destes pacientes para resolução cirúrgica, um paciente seguiu para acompanhamento ambulatorial. A retenção da coledocolitíase após um primeiro procedimento de CPRE implica novas sessões endoscópicas, uma variedade de tipos diferentes de litotripsia para reduzir o tamanho das pedras, pacientes encaminhados para cirurgia ou método paliativo, como inserção de prótese plástica biliar. ${ }^{7}$

Quatro (4) pacientes apresentaram pancreatite pós CPRE, sendo um (1) caso evoluindo a óbito, complicações estas não discordantes com as mais recentes metanálises, que demonstra taxas semelhantes destas complicações em séries maiores de 
pacientes. Não existiram perfurações ou hemorragias. Não houve nenhuma associação dos fatores avaliados, com a presença de complicações.

A CPRE é uma técnica minimamente invasiva e um tratamento com altas taxas de efetividade na retirada de cálculo (s) do ducto biliar, embora o potencial para eventos adversos sérios também seja reconhecido. Cerca de 500.000 CPREs são realizadas anualmente nos EUA com taxas de eventos adversos entre $4 \%$ e $10 \%$ e mortalidade entre $0,05 \%$ e $1 \%$, sendo os eventos adversos mais comuns são pancreatite aguda, sangramento, perfuração e sepse biliar, que ocorreram em $4 \%$ a $7 \%$ dos procedimentos. ${ }^{13}$

A pancreatite pós CPRE é o evento adverso sério mais comumente atribuído ao procedimento, em uma recente metanálise, relatou uma incidência geral de pancreatite pós CPRE de 9,7\% com aumento da incidência para 14,7\% em pacientes de alto risco (suspeita de disfunção do esfíncter de oddi, pancreatite pós CPRE prévia, sexo feminino, pacientes jovens, níveis séricos de bilirrubinas normais, dificuldade na canulação, canulação pancreática repetitiva com fio-guia, esfincterotomia pancreática, injeção pancreática de contraste e a dilatação da papila com balão de grande diâmetro em papila íntegra). ${ }^{12}$ A taxa de mortalidade por eventos adversos é inferior a $1 \%{ }^{8}$

O estudo apresentou algumas limitações, algumas próprias do modelo de estudo escolhido sendo transversal, retrospectivo, analítico e descritivo. Fatores avaliados demonstraram resultados divergentes ao já descritos na literatura, acreditamos que se deve ao número de pacientes na amostra, sendo necessário o aumento do número de casos para se confirmar ou não esta hipótese.

Apesar da alta taxa de sucesso (89\%) na extração dos cálculos, por se tratar de um hospital universitário vinculado ao SUS, há dificuldade no financiamento para aquisição de novas tecnologias e novos materiais que pudessem reduzir

\section{REFERÊNCIAS}

1. Meine GC, Baron TH. Endoscopic papillary large-balloon dilation combined with endoscopic biliary sphincterotomy for the removal of bile duct stone. Gastrointest Endosc. 2011;74(5):111926.

2. Santos JS, Sankarankutty A, Salgado W Júnior, kemp R, Módena $\mathrm{JL}$, Elias Júnior $\mathrm{J}$, et al. Colecistectomia: aspectos técnicos e indicações para o tratamento da litíase biliar e das neoplasias. Medicina (Ribeirão Preto). 2008;41(4):449-64.

3. Magalhães J, Rosa B, Cotter J. Endoscopic retrograde cholangiopancreatography for suspected choledocholithiasis: from guidelines to clinical practice. World J Gastrointest Endosc. 2015;7(2): 128-34.

4. Macedo PB Filho. Formas atuais do tratamento da coledocolitíase [Monografia]. São Paulo: Instituto Sírio Libanês de Ensino e Pesquisa; 2015 [acesso em: 19 out 2018]. Disponível em: https:// as nossas taxas de insucesso. Atualmente, a combinação entre a colangioscopia e litotripsia eletro-hidráulica, onde os principais benefícios da colangioscopia são a avaliação das estenoses e o tratamento de grandes cálculos da via biliar comum, nos quais a litotripsia eletro-hidráulica é a principal aplicação terapêutica, permitiu o tratamento da coledocolitíase com a fragmentação da pedra sob visão direta e subsequente remoção de fragmentos. ${ }^{14}$ Alguns estudos indicam taxa de sucesso da viabilidade da colangioscopia de até $97 \%{ }^{14}$

Entretanto quatro (4) pacientes (7\%) evoluíram com pancreatite pós CPRE, taxa que está em concordância com outros estudos já citados na literatura, o serviço de endoscopia do HUWC-UFC ainda não padronizou um protocolo específico para profilaxia de pancreatite pós CPRE, acreditamos que ao implementar estas medidas, será possível reduzir os eventos adversos.

A sociedade europeia de endoscopia gastrointestinal (ESGE) já recomenda a administração retal de rotina de $100 \mathrm{mg}$ de diclofenaco ou indometacina imediatamente antes ou após a CPRE em todos os pacientes que não possuam alguma contraindicação. ${ }^{15}$ Além disso, no caso de alto risco para pancreatite pós-CPRE, a colocação profilática de prótese pancreática 5-Fr deve ser amplamente considerada. ${ }^{15}$ Mok et al. (2017) descreve que em doentes com elevado risco de pancreatite, a solução de ringer lactato associado ao uso da indometacina via retal, reduziram a incidência de pancreatite pós CPRE e as taxas de readmissão em comparação com a infusão de solução salina com placebo. ${ }^{16}$

O estudo demonstra que houve alta taxa de sucesso, com taxa de complicação próximo ao já descrito na literatura, sendo a presença de cálculos grandes e a desproporção fatores relacionados ao insucesso da CPRE. Sugerindo assim a necessidade de formulação e implementação de um protocolo de medidas para profilaxia de pancreatite pós CPRE, medidas estas já recomendada e aceitas pelas principais sociedades de endoscopia.

pt.scribd.com/document/297012471/FormasAtuais-de-Tratamentode-Coledocolitiase.

5. Ortigara L, Almeida G, Araújo LF, Guerra E. Avaliação préoperatória dos pacientes com coledocolitíase. Revista Técnico Cientifica do Grupo Hospitalar Conceição. 2005;18(1):8-16.

6. Rego AC, Nunes N, Pereira JR, Paz N, Duarte MA. Dilatação papilar com balão de grande diâmetro precedida de esfincterotomia para remoção de cálculos da via biliar principal: casuística de um ano. J Port Gastrenterol. 2011;18(6):279-84.

7. García-Cano J, Arana LT, Ayllón CJ, Chicano MV, Fernández RM, Sánchez LS, et al. Biliary sphincterotomy dilation for the extraction of difficult common bile duct stones. Rev Esp Enferm Dig. 2009;101(8):541-5.

8. Chandrasekhara V, Khashab MA, Muthusamy VR, Acosta RD, 
Agrawal D, Bruining DH, et al. Adverse events associated with ERCP. Gastrointest Endosc. 2017;85(1):32-47.

9. Tantau M, Mercea V, Crisan D, Tantau A, Mester G, Vesa S, et al. ERCP on a Cohort of 2,986 Patients with cholelitiasis: a 10year experience of a single center. J Gastrointestin Liver Dis. 2013;22(2):141-7.

10. Guo SB, Meng H, Duan ZJ, Li CY. Small sphincterotomy combined with endoscopic papillary large balloon dilation vs sphincterotomy alone for removal of common bile duct stones. World J Gastroenterol. 2014;20(47):17962-9.

11. Ersoz G, Tekesin O, Ozutemis O, Gunsar F. Biliary sphincterotomy plus dilation with a large balloon for bile duct stones that are difficult to extract. Gastrointest Endosc. 2003;57(2):156-9.

12. Willian E, Beckingham I, El Sayed G, Gurusamy K, Sturgess R, Webster G, et al. Updated guideline on the management of common bile duct stones (CBDS). Gut. 2017;66:765-82.
13. Coelho-Prabhu N, Shah ND, Van Houten H, Kamath PS, Baron TH. Endoscopic retrograde cholangiopancreatography: utilisation and outcomes in a 10-year population-based cohort. BMJ Open. 2013;3(5):e002689.

14. Moura H, Franzinni T, Moura R, Carneiro F, Artifon E, Sakai P. Colangioscopia nas afecções biliares: uma série de casos. Arq Gastroenterol. 2014;51(3):250-4.

15. Dumonceau JM, Andriulli A, Elmunzer B, Mariani A, Meister T, Deviere J, et al. Updated ESGE Guideline for PEP prophylaxis. Endoscopy. 2014;46:799-815.

16. Mok S, Ho C, Shah P, Patel M, Gaughan J, Elfant A. Lactated ringer's solution in combination with rectal indomethacin for prevention of post-ERCP pancreatitis and readmission: a prospective randomized, double-blinded, placebo-controlled trial. Gastrointest Endosc. 2017;85(5):1005-13.

\section{Como citar:}

Matos DC, Tavares PR, Benevides GM, Costa LJ, Carneiro FO, Nobre e Souza MA, et al. Colangiopancreatografia retrógrada endoscópica por coledocolitíase no Hospital Universitário Walter Cantídio - UFC - avaliação de 56 casos. Rev Med UFC. 2020 jan-mar;60(1):22-27. 